ORIENTAL JOURNAL OF
ISSN: 0974-6471
March 2017,
Col. 10, No. (1):
Pgs. 160-167

\title{
A Novel Blind Digital Watermarking Based on SVD and Extreme Learning Machine
}

\author{
NEELAM DABAS ${ }^{1}$, RAMPAL SINGH$^{2}$ and VIKASH CHAUDHARY ${ }^{3}$ \\ ${ }^{1}$ Computer Science Department, University of Delhi, Delhi, India. \\ ${ }^{2}$ Computer Science Department, DDUC University of Delhi, Delhi, India. \\ ${ }^{3}$ Computer Science Department, BNC University of Delhi, Delhi, India. \\ ${ }^{*}$ Corresponding author E-mail: ansingh.du@gmail.com
} http://dx.doi.org/10.13005/ojcst/10.01.21

(Received: March 05, 2017; Accepted: March 16, 2017)

\begin{abstract}
Modification of media and illegal production is a big problem now a days because of free availability of digital media. Protection and securing the digital data is a challenge. An Integer Wavelet Transformation (IWT) domain based robust watermarking scheme with Singular Value Decomposition (SVD) and Extreme Learning Machine (ELM) have been proposed and tested on different images. In this proposed scheme, a watermark or logo is embedded in the IWT domain as ownership information with SVD and ELM is trained to learn the relationship between the original coefficient and the watermarked one. This trained ELM is used in the extraction process to extract the embedded logo from the image. Experimental results show that the proposed watermarking scheme is robust against various image attacks like Blurring, Noise, Cropping, Rotation, Sharpening etc. Performance analysis of proposed watermarking scheme is measured with Peak Signal to Noise Ratio (PSNR) and Bit Error Rate (BER).
\end{abstract}

Keywords: IWT, SVD, ELM, PSNR, BER.

\section{INTRODUCTION}

With the invention and expansion of internet, data in digital form is distributed and copied easily worldwide. But with the distribution, protection or security of data is equally important. Watermarking is an emerging technique for the security of data. Watermarking is the process that embeds data called a watermark, tag or label into a multimedia object such that watermark can be detected or extracted later to prove the ownership ${ }^{1}$. Its applications include broadcast monitoring, data authentication, protection of ownership etc ${ }^{1}$ Over the past years, many singular value decomposition (SVD) based watermarking schemes are proposed ${ }^{2,3,4}$, in which three matrices are modified slightly to embed the watermark. Later, all these SVD based watermarking algorithms are extended to embed the watermark in wavelet domains to provide better robustness ${ }^{5}$. We are proposing a method with the combination of SVD and Extreme Learning Machine (ELM) in Integer Wavelet Domain (IWT). ELM is an algorithm for single layer feed forward neural network, where parameters of neural network like weights and bias are randomly selected. Training time of ELM is very 
fast since weights and bias are not adjusted by using gradient descent method 6 . Gradient descent method have the problem of slow learning rate, local minima etc. IWT domain reduces the signal loss during the inverse process.

Rest of the paper is organized as follows. We give some background theories about IWT, SVD and ELM in Section 2. Proposed water marking schemes watermark embedding, ELM training and watermark extraction are described in Section 3.

Experimental results are discussed in Section 4 followed by conclusion in Section 5 .

\section{Literature Surcey Of Iwt, Svd And EIm Integer Wavelet Transform (IWT)}

To increase the robustness, watermarks are to be embedded in wavelet domain instead of spatial domain. The image is divided into low and high resolution bands ( $L L, H L, L H, H H)$. In discrete wavelet transform, we hide data into floating point coefficients, so during the inverse transformation, any truncation in floating point value leads to the loss of information. IWT transforms a data set into another integer data set $^{7}$. So during forward and inverse transformation, no loss of information is there which leads to have a very close copy of original image ${ }^{8}$. Lifting schemes are used to perform IWT. IWT process is divided into three steps ${ }^{9}$.

1. Split: Partition the data set $\beta_{\mathrm{j}}$ into low and high frequency samples.

Split $\left(\beta_{\mathrm{j}}\right)=\left\{\operatorname{odd}\left(\beta_{\mathrm{j}}\right)\right.$, even $\left.\left(\beta_{\mathrm{j}}\right)\right\}=\left\{\gamma_{\mathrm{j}}, \gamma_{\mathrm{j}}\right\}$

2. Predict: Predict the odd elements $\gamma_{j}$ from the even elements $\lambda_{i}$

3. Update: Update the data in the set with the data in the set.

\section{Singular Value Decomposition (SVD)}

In linear algebra, SVD is a technique to factorize a rectangular matrixinto three decomposition matrices

$$
\mathrm{I}_{\mathrm{m} \times \mathrm{n}}=\mathrm{U}_{\mathrm{m} \times \mathrm{m}} \mathrm{D}_{\mathrm{m} \times \mathrm{n}} \mathrm{V}_{\mathrm{n} \times \mathrm{n}}^{\top}
$$

$=\left[u_{1}, u_{2}, \ldots . u_{m}\right] *\left(\begin{array}{ccc}d_{1} & 0 & 0 \\ 0 & d_{2} & 0 \\ 0 & 0 & d_{n}\end{array}\right) *\left[v_{1}, v_{2}, \ldots . v_{n}\right]$
By multiply $U, D, V^{\top}$, we can get the matrix

lback.

$I_{m \times n}=U_{m \times m} * D_{m \times n} * V_{n \times n}^{T}$

The diagonal entries $d_{1}, d_{2}, \ldots, d_{n}$ in diagonal matrix $D$ are called the singular values. They are related with the image luminance while the $U$ and $V$ the horizontal and vertical details of image determine the "geometry" of the image ${ }^{10}$. SVD is a popular method for image watermarking algorithm as singular values are robust against various common image processing operations and geometric transformations like scaling, rotation, translation etc ${ }^{11}$.

\section{Extreme Learning Machine (ELM)}

ELM algorithm is proposed by Huang et al. ${ }^{12}$ which is based on SLFNs. ELM overcomes the pitfalls of Artificial Neural Network like local minima and slow learning rate. Consider a SLFN with $N$ input layer, $M$ output layer with $L$ hidden neuron. Take $N$ samples where is vector and is M output vector. Randomly select two parameters, bias and weights where is the weight vector of the connection between $i^{\text {th }}$ input layer and $k^{\text {th }}$ hidden neuron of input layer. The output function $T$ with activation function is given by

$T_{L}(x)=\sum_{i=1}^{L} \beta_{i} g_{i}(x)=\sum_{i=1}^{L} \beta_{i} G\left(w_{i}, b_{i}, x\right)=o_{i}$

Where is the weight vector connecting the $i^{\text {th }}$ hidden node and the output nodes, $b_{i}$ is the threshold value of $i^{\text {th }}$ hidden nodes. For additive hidden nodes the activation function is defined as

$G\left(w_{i}, b_{i}, x\right)=g\left(w_{i} \cdot x+b_{i}\right)$

Where denotestheinnerproductofvector and $x$ in . For an RBF hidden node the activation function $g(x)$ is given by

$G\left(w_{i}, b_{i}, x\right)=g\left(b_{i}\left\|x-w_{i}\right\|\right)$

Where $w_{i}$ and $b_{i}$ are the center and the impact factor of the $i^{\text {th }}$ RBF node. Equaion (4) can be re-written as

$H \beta=T$ 
Where $H$ is the hidden layer output matrix and $T$ is the target vector. are estimated as

$$
\beta=\mathrm{H}^{\dagger} \mathrm{T}
$$

Where is the Moore-Penrose generalized pseudo inverse ${ }^{13}$.

\section{Essence of ELM}

The basic essence of ELM is that:

1) No iterative tuning is required in SLFN,, , parameters of hidden layer are randomly chosen $^{12},{ }^{14}$.

2) $\quad \mid \mathrm{H} \beta-\mathrm{T} \|$, the training error and, norm of output weight need to be minimized ${ }^{12},{ }^{14},{ }^{15}$.

3) Least square method is used to calculate between the hidden layer and the output layer $^{16,6}$

\section{Algorithm}

ELM algorithm is as follows: For $N$ training samples $\left(x_{i}, t_{i}\right) \in R^{N} \times R^{M}, L$ number of hidden neurons and $g(x)$ as an activation function

1) Input weight $W_{i}$ and bias $b_{i}$ are randomly generated, where $\mathrm{i}=1, \ldots, \mathrm{N}$

2) Calculate $H$, hidden neuron output matrix.

3) Calculate $\beta$, output weight using equation

$$
\beta=\mathrm{H}^{\dagger} \mathrm{T}
$$

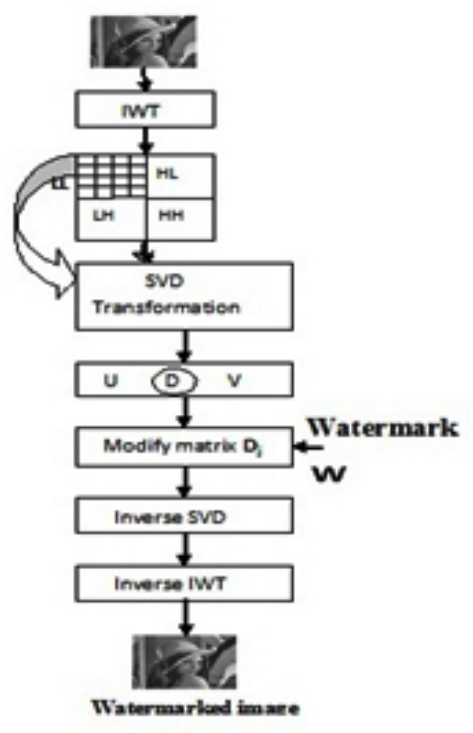

Fig.1: Proposed Watermark embedding process

\section{Proposed Watermarking Scheme}

This algorithm consists of three parts: Watermark embedding, ELM Training and Watermark extraction

\section{Watermark Embedding}

The block diagram of watermark embedding is shown in Fig(1).

Let us assume there is a host image $H$, of size and a binary watermark image $w$ with size. As it is a binary image so. In our case, size of image $H$ is and watermark wis. The embedding algorithm is as follows:

1. Host image $H$ is transferred through 1-level IWT to decomposed it into $(L L, L H, H L, H H)$ sub-bands.

2. Out of these four sub-bands $(L L, L H$, $H L, H H$ ), $L L$ (Lowest level) has been selected for watermark embedding as it contains maximum energy. Now $L L_{i j}$ sub-band is partitioned into $4 \times 4$ non-overlapping coefficient blocks.

3. Apply $S V D$ on each blocks to get three components, $U_{i j} D_{i j}$ and $V_{i j}$

$$
L L^{i j}=\bigcup_{i j}^{i j} D_{i j} V_{i j}{ }^{i j}
$$

4. Take out the four singular values of each $L L_{i j}$

$$
D_{\mathrm{ij}}=\left(\mathrm{d}_{1}, \mathrm{~d}_{2}, \mathrm{~d}_{3}, \mathrm{~d}_{4}\right)
$$

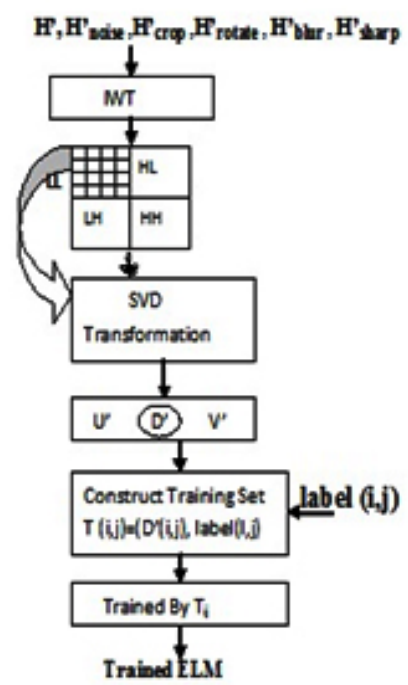

Fig.2. ELM Training Process 
5. Modify the values of $d_{2}$ based on the following mathematical operations where $\alpha$ is embedding strength of watermarking scheme

$$
\begin{gathered}
d_{2}=\left\{\begin{array}{l}
\mu+\alpha \times \delta \text {. if } w_{i j}=1 \text { and } d_{2}<\mu \\
d_{2} \quad \text { if } w_{i j}=1 \text { and } d_{2}>\mu \\
\mu-\alpha \times \delta \text {. if } w_{i j}=0 \text { and } d_{2}>\mu \\
d_{2}, \quad \text { if } w_{i j}=0 \text { and } d_{2}<\mu
\end{array}\right. \\
\mu=\frac{1}{4} \times \sum_{i=1}^{4} d_{i}
\end{gathered}
$$

6. Perform inverse SVD to get the modified coefficient block.

$$
\mathrm{U}_{\mathrm{ij}} \mathrm{D}_{\mathrm{ij}}^{\prime} \mathrm{V}_{\mathrm{ijT}}=\mathrm{LL}_{\mathrm{ij}}^{\prime}
$$

7. Perform inverse IWT with $\mathrm{LL}_{\mathrm{ij}} \mathrm{HL}, \mathrm{LH}, \mathrm{HH}$ to get the final watermarked image $H^{\prime}$.

\section{ELM Training}

The block diagram to train an ELM is shown in fig (2).

Training set is derived from the original watermarked image $H^{\prime}$ and the corrupted watermarked images by Gaussian noise $H_{\text {noise' }}$,

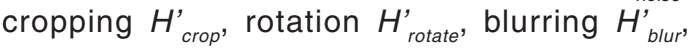
sharpening $H_{\text {sharp }}^{\prime}$ respectively. Training of ELM is as follows:

1. Apply IWT on $H^{\prime}, H_{\text {noise, }}^{\prime} H_{\text {crop, }}^{\prime} H_{\text {rotate, }}^{\prime} H_{\text {blur, }}^{\prime} H_{\text {sharp }}^{\prime}$ and get their respective $L L$ sub-bands denoted $\operatorname{as} L L^{\prime}, L L_{\text {noise, }}^{\prime}, L L_{\text {crop }}^{\prime}, L L_{\text {rotate }}^{\prime}, L L_{\text {blur' }}^{\prime} L L_{\text {sharp }}^{\prime}$

2. Each $L L$ sub-band ( $L L^{\prime}, L L_{\text {noise }}^{\prime}, L L_{\text {crop }}^{\prime}$, $\left.L L_{\text {rotate }}^{\prime}, L L_{\text {blur }}^{\prime}, L L_{\text {sharp }}^{\prime}\right)$ is divided into nonoverlapping coefficient blocks.

3. Apply SVD on each blocks to get the singular values of each image $D_{i j}=\left(d_{1}, d_{2}, d_{3}, d_{4}\right)$

4. Take each sample label according to the following equation

$$
\text { label }_{i j}=\left\{\begin{array}{l}
1 \text { if } W_{i j}=1 \\
2 \text { if } W_{i j}=0
\end{array}\right.
$$

5. Construct the training set with feature vector $D_{i j}$ and label label

$\mathrm{T}_{\mathrm{ij}}^{\mathrm{k}}=\left(\mathrm{D}_{\mathrm{ij}}\right.$, (abel $\left.\mathrm{ibj}_{\mathrm{ij}}\right), \mathrm{k}=1, . .6$ and $\mathrm{i} \leq \mathrm{N}$ and $\mathrm{j} \leq \mathrm{N}$

6. Train the ELM with the training set $\mathrm{T}^{\mathrm{k}}{ }_{\mathrm{ij}}$.

\section{Watermark Extraction}

The block diagram to extract the watermark is shown in fig (3)
The process is as follows:

1. Apply IWT on watermarked image $\mathrm{H}^{\prime}$ to get LL' sub-band.

2. Partition LL' sub-band into $4 \times 4$ nonoverlapping coefficient blocks.

3. Apply SVD on $L L^{\prime}$ ij to get the feature vector $D^{\prime}{ }_{i j}=\left(d_{1}{ }_{1}, d^{\prime}{ }_{2}, d^{\prime}{ }_{3}, d^{\prime}{ }_{4}\right)$

4. By using well trained ELM, get the predicted label label $l_{i j}$, corresponding to each $D_{i j}^{\prime}$.

5. Watermark can be extracted by using the predicted label as

$$
W_{i j}^{\prime}=\left\{\begin{array}{l}
1 \text { if label } l_{i j}^{j}=1 \\
0 \text { if label } l_{i j}^{\prime}=2
\end{array}\right.
$$

\section{Experimental Results For Robustness Of The Proposed Watermarking Scheme}

In this paper, an experiment is performed on host images like Lena, Baboon, Pepper, Elaine and Jet of size $512 \times 512$ and a watermark logo of size $32 \times 32$ is used. The value of $\propto$ is taken as 0.3 Performance of watermarking algorithm is done on the basis of two parameters imperceptibility and robustness. PSNR is used to measure the quality of watermarked image with the original host image. Higher the value of PSNR, better is the quality of watermarked images. PSNR between the original image and watermarked image $\mathrm{H}$ and $\mathrm{H}^{\prime}$ is ${ }^{17}$.

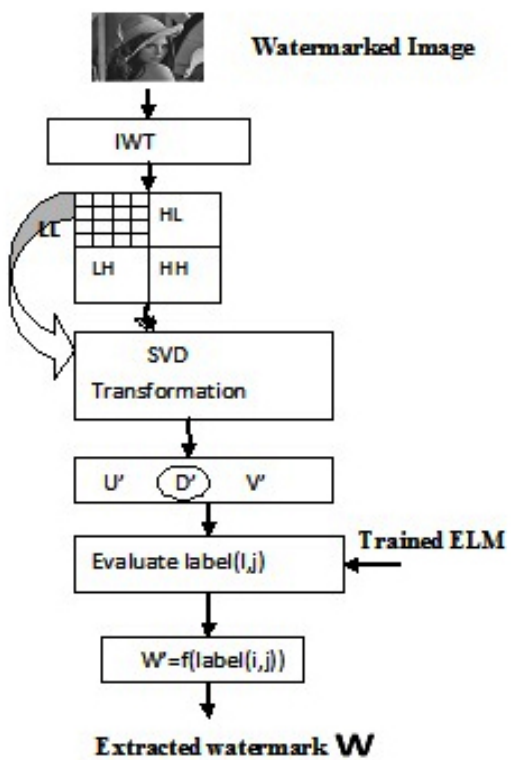

Fig. 3: Proposed Watermark Extraction process 
$P S N R=10 \log \log _{10} \frac{255 \times 255}{\sum_{i=0}^{m-1} \sum_{j=0}^{n-1}\left(x_{i j}-x_{i j}^{\prime}\right)^{2}} /(m \times n)$

and BER is evaluated as

$B E R=\sum_{t=1}^{p x q}\left(w_{t} \oplus w_{t}^{\prime}\right) / p x q$

where $w_{t}$ is original watermark and $w_{t}^{\prime}$ is the extracted watermark. $p \times q$ is the size of

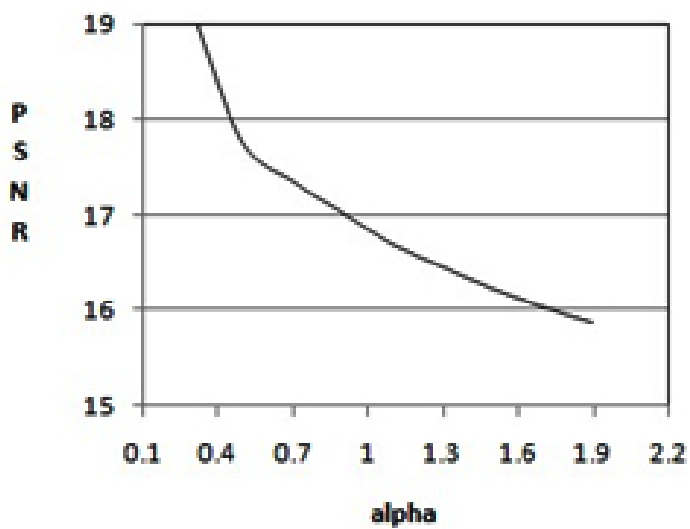

Fig.4: Relation between PSNR and $\propto$. watermark and $\oplus$ is an exclusive-OR operator Lower the value of BER implies greater similarity between the extracted watermark and the original one.

The value of $\propto$ is tested in the interval $\propto \in[0,0.3]$. It is found that the value of PSNR is decreasing with the value of $\propto$ as shown in Fig(4).

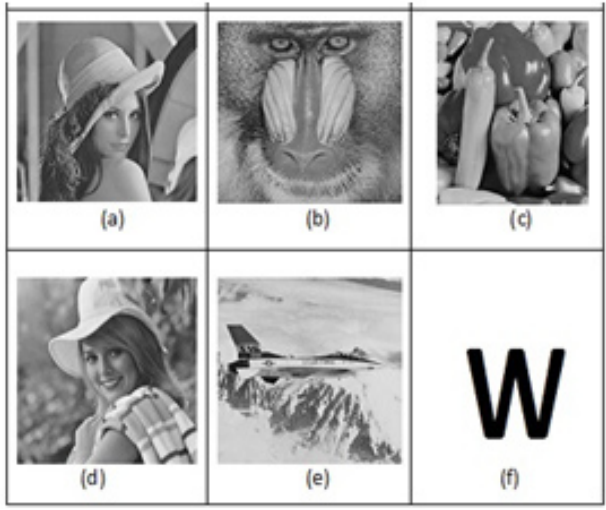

Fig.5: (a) Lena (b) Baboon (c) Peppers (d) Elaine (e) Jetplane images used for watermarking and (f) the Watermark logo

Table 1: Attacked Lena images, corresponding value of BER, PSNR and extracted binary watermark (ownership) from attacked watermarked Lena image by using the proposed watermarking scheme

\begin{tabular}{|c|c|c|c|c|}
\hline Attacks & Image & BER & PSNR & Ownership \\
\hline Blurring & & 0.0205 & 23.06 & \\
\hline Cropping & & 0.1074 & 21.70 & \\
\hline Noise & & 0.0218 & 18.7039 & \\
\hline Rotation & & 0.1441 & 17.4914 & \\
\hline Sharpening & & 0.0469 & 18.0145 & \\
\hline
\end{tabular}


Table 2: Attacked Baboon images, corresponding value of BER, PSNR and extracted binary watermark (ownership) from attacked watermarked Baboon image by using the proposed watermarking scheme

\begin{tabular}{|c|c|c|c|c|}
\hline Attacks & Image & BER & PSNR & Ownership \\
\hline Blurring & & 0.0332 & 19.3670 & \\
\hline Cropping & & 0.0850 & 21.3491 & \\
\hline Noise & & 0.0928 & 18.2904 & \\
\hline Rotation & & 0.1001 & 15.3847 & \\
\hline Sharpening & & 0.0329 & 14.2370 & \\
\hline
\end{tabular}

Table 3: Attacked Peppers images, corresponding value of BER, PSNR and extracted binary watermark (ownership) from attacked watermarked Peppers image by using the proposed watermarking scheme

\begin{tabular}{|c|c|c|c|c|}
\hline Attacks & Image & BER & PSNR & Ownership \\
\hline Blurring & & 0.0405 & 27.6210 & \\
\hline Cropping & & 0.0947 & 27.2009 & \\
\hline Noise & & 0.0752 & 23.5349 & \\
\hline Rotation & & 0.1121 & 21.5885 & \\
\hline Sharpening & & 0.0596 & 23.6203 & \\
\hline
\end{tabular}


Table 4: Attacked Elaine images, corresponding value of BER, PSNR and extracted binary watermark (ownership) from attacked watermarked Elaine image by using the proposed watermarking scheme

\begin{tabular}{|c|c|c|c|c|}
\hline Attacks & Image & BER & PSNR & Ownership \\
\hline Blurring & & 0.0869 & 23.8083 & \\
\hline Cropping & & 0.0215 & 17.9941 & \\
\hline Noise & & 0.0113 & 16.9005 & \\
\hline Rotation & & 0.1022 & 16.1664 & \\
\hline Sharpening & & 0.0669 & 15.1334 & \\
\hline
\end{tabular}

Table 5: Attacked Jetplane images, corresponding value of BER, PSNR and extracted binary watermark (ownership) from attacked watermarked Jetplane image by using the proposed watermarking scheme

\begin{tabular}{|c|c|c|c|c|}
\hline Attacks & Image & BER & PSNR & Ownership \\
\hline Blurring & & 0.0415 & 25.9971 & \\
\hline Cropping & & 0.1055 & 25.6287 & \\
\hline Noise & & 0.0635 & 23.0474 & \\
\hline Rotation & & 0.1078 & 20.1516 & \\
\hline Sharpening & & 0.0613 & 23.2219 & \\
\hline
\end{tabular}




\section{CONCLUSIONS}

In this paper, we proposed a novel combination of IWT, SVD and ELM for authentication of ownership. In the proposed scheme, host image is transformed in IWT domain and then LL subband is used to take the singular values, where required numerical operations are done to embed the watermark. Watermark extraction is a two-step process, firstly training of ELM and secondly the actual watermark extraction for proof of ownership. As shown in experimental results, our proposed method is robust against various attacks and the extracted watermark, to prove the ownership is very much similar to the original watermark, i,e less BER value.

\section{REFERENCES}

1. S.P. Mohanty, "Digital Watermarking: A tutorial review," 1999.

2. Piyu Tsai, Chia-Chen Lin Chin-Chen Chang, "SVD-based digital image watermarking scheme," Pattern Recognition Letters, 2005.

3. R.Liu and T.Tan, "An SVD-based watermarking scheme for protecting rightful ownership," IEEE Trans. Multimedia, 2002.

4. D.V.S. Chandra, "Digital image watermarking using singular value decomposition," Proc. of the The 45th Midwest Symposium on Circuits and Systems (MWSCAS)., 2002.

5. $\mathrm{P}$ Bao and $\mathrm{X} \mathrm{Ma,} \mathrm{"Image} \mathrm{adaptive}$ watermarking using wavelet domain singular value decomposition," IEEE Trans. Circuits and Systems for Video Technology, 15, pp. 96-102, 2005.

6. G.B.Huang, Q.Y.Zhu and C.K.Siew , "Extreme learning machine: theory and applications," Neuro computing, 70, pp. 489501, 2006.

7. A.W.Bojanczyk, et al., "Wavelet transforms that map integers to integers.," Appl. Comput. Harmon. Anal., 5(3), pp. 332-369., 1998.

8. M. Ramani, Dr. E. V Prasad, and Dr. S. Varadarajan, "Steganography Using BPCS the Integer Wavelet Transformed bnage," UCSNS International Journal of Computer Science and Network Security, 7(7), July 2007.

9. S. Lee, C.D. Yoo and T. Kalker, "Reversible image watermarking based on integer-tointeger wavelet transform," IEEE Transactions on Information Forensics and Security,2(3), pp. 321-330, Sep 2007.

10. A.W. Bojanczyk, 0 , "An accurate product SVD algorithm.," Signal Process., 25(2), pp. 189-201, 1991.

11. L.L.Scharf, "The SVD and reduced rank signal processing.," Signal Process., 25(2), pp. 113-133, 1991.

12. G-B. Huang, D-H. Wang and Y. Lan , "Extreme Learning Machine : A Survey," International Journal of Machine Learning and Cybernitics, 2, pp. 107-122, 2011.

13. C.R.Rao and S.K.Mitra, "Generalized Inverse of Matrices and its Applications," Wiley, New York, 1971.

14. G-B Huang, L. Chen L, C-K.Siew, "Extreme learning machine: a new learning scheme of Feedforward networks," , 2, Budapast, Hungary, 2004, pp. 985-990.

15. P.L. Bartlett, "The sample complexity of patten classification with neural networks:The size of weights is more important than the size of the network," IEEE Trans. of Information Theory, 44, pp. 525-536, 2006.

16. Huang G-B, Chen L, Siew C-K, "Universal approximation using incremental constructive feedforward networks with random hidden nodes.," IEEE Trans Neural Netw, 17(4), pp. 879-892, 2006.

17. Jinkou Ding, Qiaoyan Wen, Xin Liao, Cuixiang Liu Ben Wang, "An Image Watermarking Algorithm Based On DWT DCT and SVD," Proceedings of IC-NIDC., 2009. 